\title{
OPEN Ultrafast electron holes in plasma phase space dynamics
}

\author{
Seyyed Mehdi Hosseini Jenab ${ }^{1,2 \bowtie}$, Gert Brodin ${ }^{3}$, James Juno ${ }^{4}$ \& loannis Kourakis ${ }^{5}$
}

Electron holes $(\mathrm{EH})$ are localized modes in plasma kinetic theory which appear as vortices in phase space. Earlier research on EH is based on the Schamel distribution function (df). A novel df is proposed here, generalizing the original Schamel df in a recursive manner. Nonlinear solutions obtained by kinetic simulations are presented, with velocities twice the electron thermal speed. Using 1D-1V kinetic simulations, their propagation characteristics are traced and their stability is established by studying their long-time evolution and their behavior through mutual collisions.

Plasma phase-space dynamics is tacitly characterized by the occurrence of electron holes, a term describing a localized plasma region where electrons are trapped by the electric potential stemming from their own selfgenerated density variation, as a localized electron depletion region occurs in a self-consistent manner. An electron hole is thus manifested as a localized "trapped" electron population traveling alongside an electrostatic potential disturbance ${ }^{1,2}$. Electron-holes present two main characteristics ${ }^{3}$ : a localized positive potential structure which traps electrons, and a symmetry in the electric potential profile around the peak. In addition, electron holes are a type of Bernstein, Greene, and Kruskal (BGK) mode ${ }^{4}$. Electron holes have been observed and studied in laboratory experiments ${ }^{5}$, in space measurements ${ }^{6-10}$ and in kinetic simulations ${ }^{11}$.

In order to construct electron holes in a self-consistent manner within a kinetic model, one may either start with an arbitrary potential profile and then proceed by deriving the distribution function (df) of an electron hole, or, inversely, start with a predefined $\mathrm{df}$ for the trapped electrons and thus derive the associated potential profile. The former (integral equation) method, due to Bernstein, Greene and Kruskal ${ }^{4}$ leads to an infinity of solutions whose dynamical stability is not prescribed. The latter (differential equation) method, suggested by Schamel ${ }^{12-15}$, is based on a parametrized $d f$ (henceforth referred to as "the Schamel df") allowing one to prescribe the shape of the trapped population (i.e. by assigning a value to parameter $\beta$ associated with the inverse temperature of the trapped population). Recently, Schamel df is extended by adding new parameters and hence resulted in variety of new solutions. Note, most of the solutions, i.e. $\phi(x)$ are undisclosed ${ }^{16}$. In the case of double layers, the Schamel df provides solutions which are much faster than the thermal velocity ${ }^{17}$. In fact, as the authors in Ref. ${ }^{17}$ have predicted, a strong double layer (DL) solution is obtained as a limiting variant of a solitary hole; see also ${ }^{1}$ for details. The Schamel method combined with the pseudopotential approach ${ }^{18}$ may provide initial conditions for a controlled numerical investigation of EH dynamics ${ }^{19}$. Recent studies ${ }^{19,20}$ have shown that the Schamelpseudopotential approach can produce nonlinear solutions with Mach numbers $1.0<M<10.0$.

However, only solutions in the range $1.0<M<3.0$ are found to be stable for long times ${ }^{19}$ and to survive mutual collision ${ }^{20}$. In other words, structures are destabilized as the Mach number increases. This has been suggested in other kinetic simulations ${ }^{21}$. For very high Mach number $(M>10)$, the Schamel-pseudopotential method can not provide any solutions even for a wide range of $\beta$ (values) ${ }^{3,19}$. The maximum speed for a soliton accompanied by an electron hole (SEH) is $M=1.307$ when using the pseudopotential appoach in the smallamplitude regime ${ }^{22}$.

Despite these theoretical challenges, the existence of high-speed electron holes is a topic of intense study, first getting attention due to observations by the FAST satellite ${ }^{6,23,24}$. Saeki et al. ${ }^{5}$ studied electron holes experimentally using a Q-plasma machine and also via kinetic simulations; they reported structures moving at the electron thermal speed, which they identified as solitons. Solitons are nonlinear structures that can survive mutual collisions and are characterized by a phase shift during a collision ${ }^{25-29}$. We note however, Saeki et al. did not consider the phase shift separating the hole trajectories before and after collisions. It is interesting to point out that fast (large Mach number) localized structures have also been predicted recently, in the form of supersolitons (supernonlinear waves); see e.g. ${ }^{30-32}$. Nonetheless, it is important to realize that these structures are distinct in both their

\footnotetext{
${ }^{1}$ Department of Physics, Chalmers University of Technology, 41296 Göteborg, Sweden. ${ }^{2}$ Department of Electrical, Computer and Biomedical Engineering, Ryerson University, Toronto M5B 2K3, Canada. ${ }^{3}$ Department of Physics, Umeå University, Umeå, Sweden. ${ }^{4}$ Department of Physics and Astronomy, University of lowa, lowa City, IA 52242, USA. ${ }^{5}$ Department of Mathematics, Khalifa University of Science and Technology, Abu Dhabi, UAE. ${ }^{\square}$ email: mehdi.jenab@chalmers.se
} 


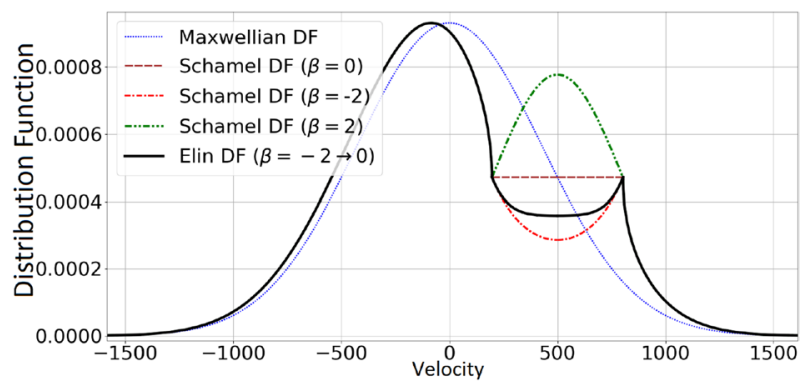

Figure 1. Different distribution functions for the trapped electron population are presented. The Maxwellian $\mathrm{df}$ (in the absence of trapped particles) is shown for sake of comparison (blue, thin dotted line). Three shapes of the Schamel df are displayed, namely flat (brown dashed, $\beta=0$ ), hollow (red, dashed-dotted, $\beta=-2$ ) and a bump (green, dashed-dotted, $\beta=2$ ), for $\phi=25$. The ELIN df (black thick line) is shown when ten carving $\left(\phi_{1}=2.5, \phi_{2}=5, \phi_{3}=7.5, \ldots, \phi_{10}=25\right)$ is carried out with their corresponding $\beta$ $\left(\beta_{1}=-2, \beta_{2}=-1.8, \beta_{3}=-1.6, \ldots, \beta_{10}=0\right)$.
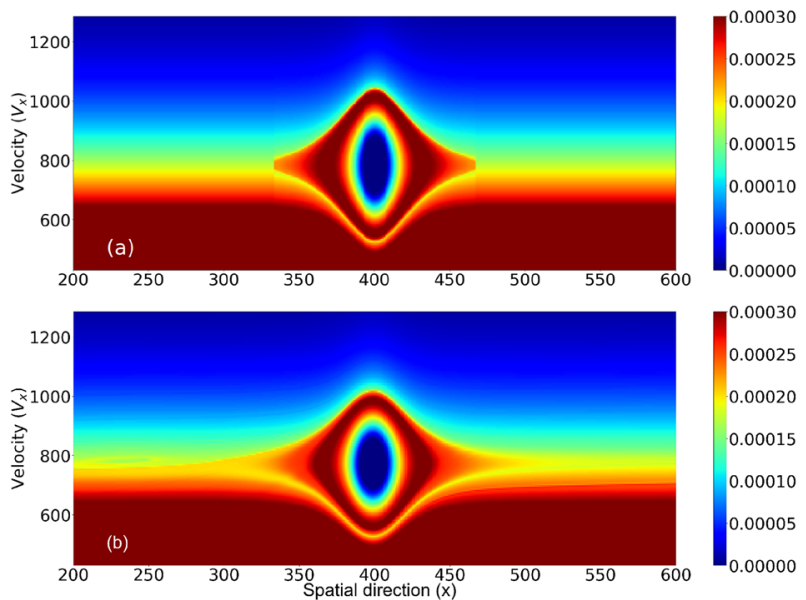

Figure 2. The electron phase space is shown for the case $E H 1(\mathbf{a})$ at the initial step and (b) at $\tau=12$.

structural characteristics (shape) and in the physical mechanism underlying their formation. (An interested reader is referred to the above references for details).

The aim of this study is to characterize high-speed electron holes by establishing their occurrence in a kinetic framework, and by investigating their stability profile and probing their soliton-like features. For this purpose, a novel distribution function $(d f)$, the 'ELIN df', is introduced as a generalization of the Schamel df. The ELIN $\mathrm{df}$ adjusts the distribution function of the trapped population of electrons by relying on a dynamically varying parameter $\beta$ so that its moments can fit a predetermined curve and all of the desired featured of the Schamel df are retained, such as consistency and smoothness in both spatial and velocity spaces inside the trapped region (Fig. 1).

To show the stability of our nonlinear solutions, three series of simulations are reported. Firstly, by considering the long-time evolution of an initial condition we will confirm the stability of the solution's profile during propagation, thus establishing them as solitary waves. Then, two types of mutual collisions are reported, i.e. head-on collisions (with no overlapping in velocity space) and overtaking collisions (moving in parallel and with overlapping). The aforementioned phase shift through collisions has also been investigated, to corroborate the fact that electron holes behave as solitons.

\section{Results}

Long-term evolution. Figures 2 and 3 display the temporal evolution of $E H 1$. The initial condition and the last step of temporal evolution can be compared and show that the overall shape of the electron hole (Fig. 2) and the corresponding potential or field profile (Fig. 3)stay unperturbed.

Head-on collision. Figure 4 depicts a head-on collision between EH1 and EH2. After the collision $(0<\tau<2)$, both solutions keep their shape and velocity compared to their initial state. Note that due to their large velocity, they are well-apart in the velocity direction, i.e. there is no overlapping, and hence their collision 

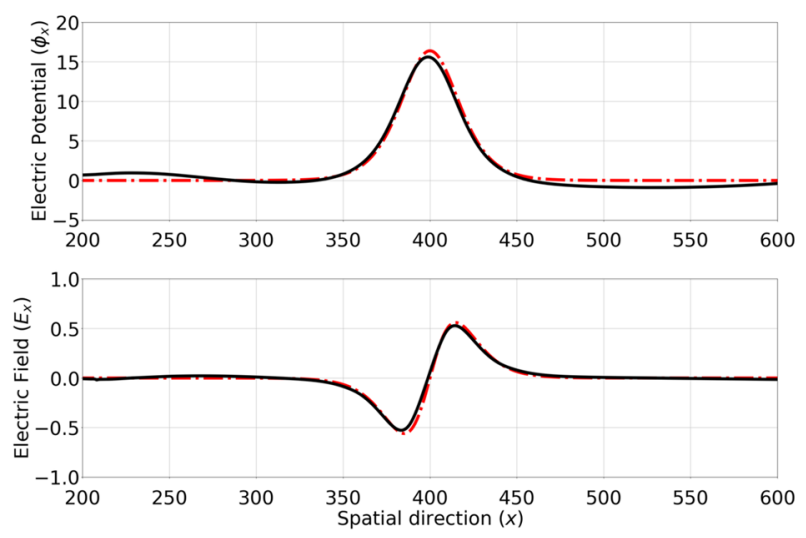

Figure 3. The electrostatic potential/E-field profile of $E H 1$ is shown in the top/bottom panel. The initial condition i.e. at $\tau=0$ (red dotted curve) is compared with $\tau=12.0$ (solid black curve), showing a good agreement and hence confirming the stability of EHs during long-time propagation.
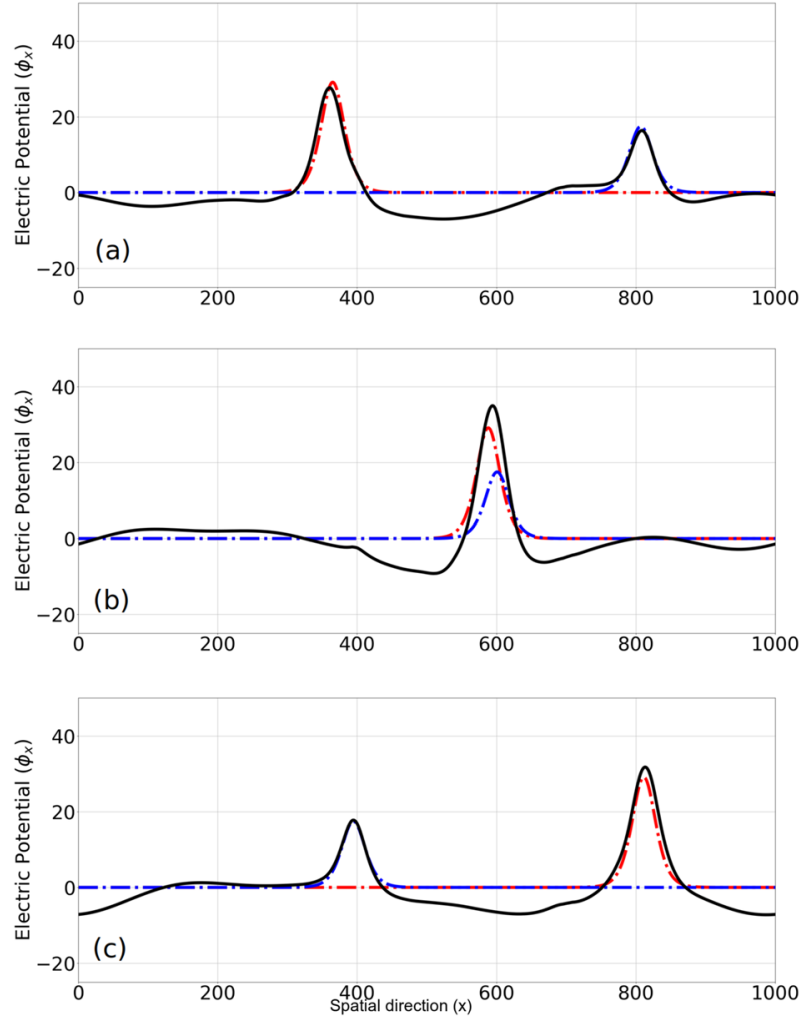

Figure 4. The electrostatic potential profile of $E H 1$ and $E H 2$ is shown at different snapshots around a head-on collision, namely (a) before ( $\tau=1.3)$, (b) during $(\tau=1.6)$ and (c) after $(\tau=1.9)$ the collision. Dotted lines represent the initial condition for each of the solitary wave as if they are propagating without any numerical noise or collisions. Red/blue is for EH1/EH2 which is propagating to the right/left. After the collision, the overall shape and velocity of the solitary wave remains intact.

on the phase space consists of two electron holes passing each other without much interaction. Both electron holes follow their unperturbed trajectories after the collision, hence no phase shift is observed.

Overtaking collision. Although the previous simulations demonstrate the stability of these EHs, the strongest test of the stability is their interaction via an overtaking collision when they overlap in the velocity direction. In an overtaking simulation, we have used two EHs e.g. $E H 1$ and $E H 3$. Figure 5 presents the temporal evolution of electric field/potential around the collision time $\tau=3.2$ in a frame moving with $M=45$. Both EHs survive 

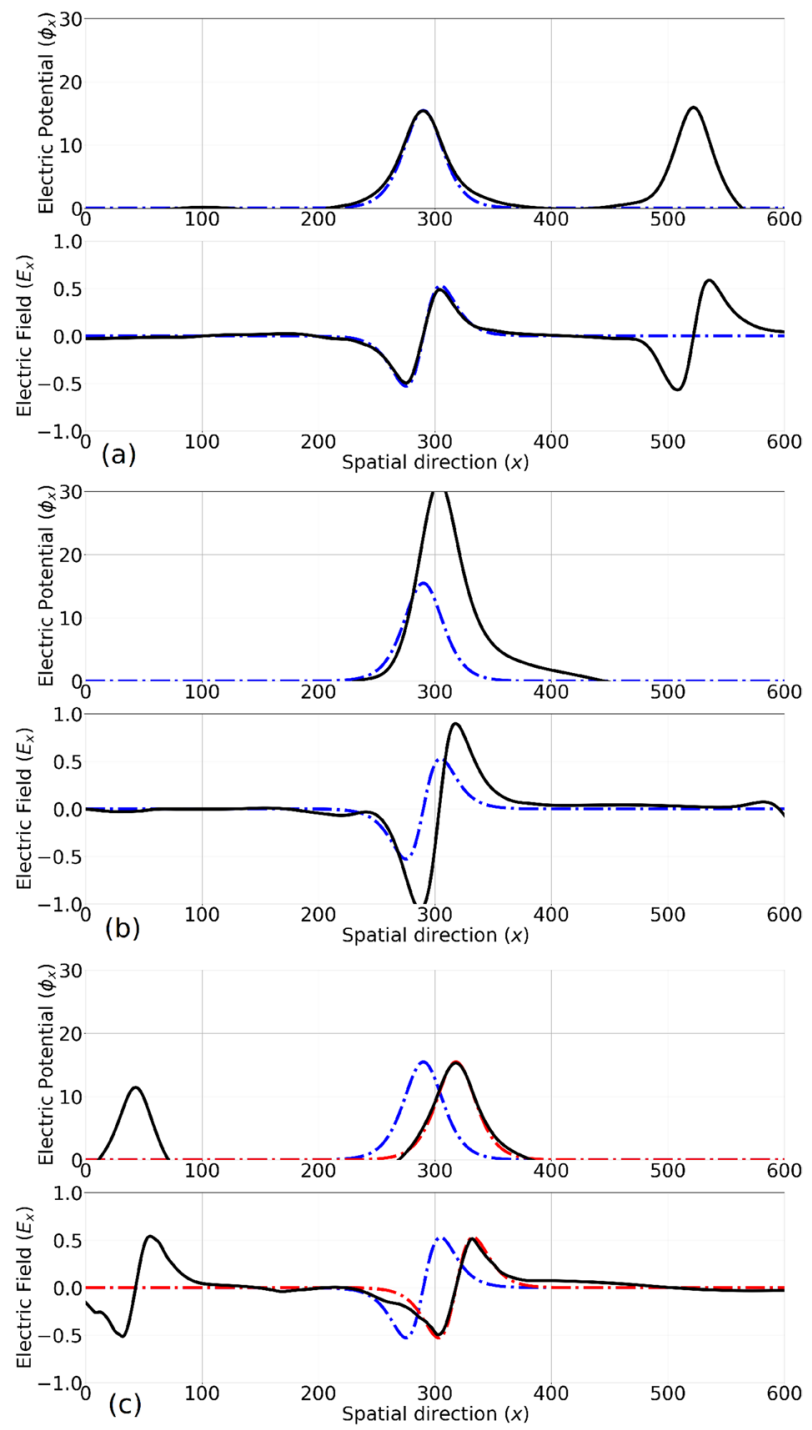

Figure 5. An overtaking collision between $E H 1$ and $E H 3$ is presented by plotting the electrostatic potential and the electric field profile in the co-moving frame of $E H 1$ at three snapshots: (a) before $(\tau=2.35)$, (b) during ( $\tau=3.20)$ and (c) after $(\tau=4.27)$ the collision. The dotted curves show the fitted profile ( sech $^{2}$ before (blue) and after (red) the collision, for EH1. A shift in the position of the first EH can be witnessed (note the difference between the red and the blue curves) manifesting a phase shift, as intuitively expected.

the collision, and their respective velocity stays the same. Focusing on $E H 1$, displacement can be witnessed after the collision. A phase shift can be measured by comparing EH profile with the red line, which is an extrapolation of an unperturbed path of this EH. This displacement is similar to the well-known effect of "phase shift" which observed to happen in mutual collisions of solitons ${ }^{25-29}$.

We show in Fig. 6 the electron df during the overtaking collision, which demonstrates the considerable interaction between the EHs during the collision and their overlapping on velocity direction. Yet after the collision the $E H 1$ is largely unperturbed, modulo the observed phase shift. Interestingly, data fitting has shown that the $\operatorname{sech}^{2}$ curve form approximates the numerical data better than any other exponent, including the (expected, arguably) $\operatorname{sech}^{4}$ form (see Eq. 39 in $^{14}$ ).

\section{Discussion}

In summary, we have provided a method to produce high-speed nonlinear solutions which move at a speed beyond the electron thermal speed. We showed that these electron holes are stable, retain their profile through collisions and remain so in the entire duration of the simulation. For mutual collisions with considerable overlap in the velocity direction, the EHs display a "phase shift" This phase shift represents a signature of soliton behavior and hence suggests that these EHs can be considered as solitons (at least approximately). This has been suggested for much lower-speed EHs before ${ }^{5}$ but without the observed "phase shift" reported here. 

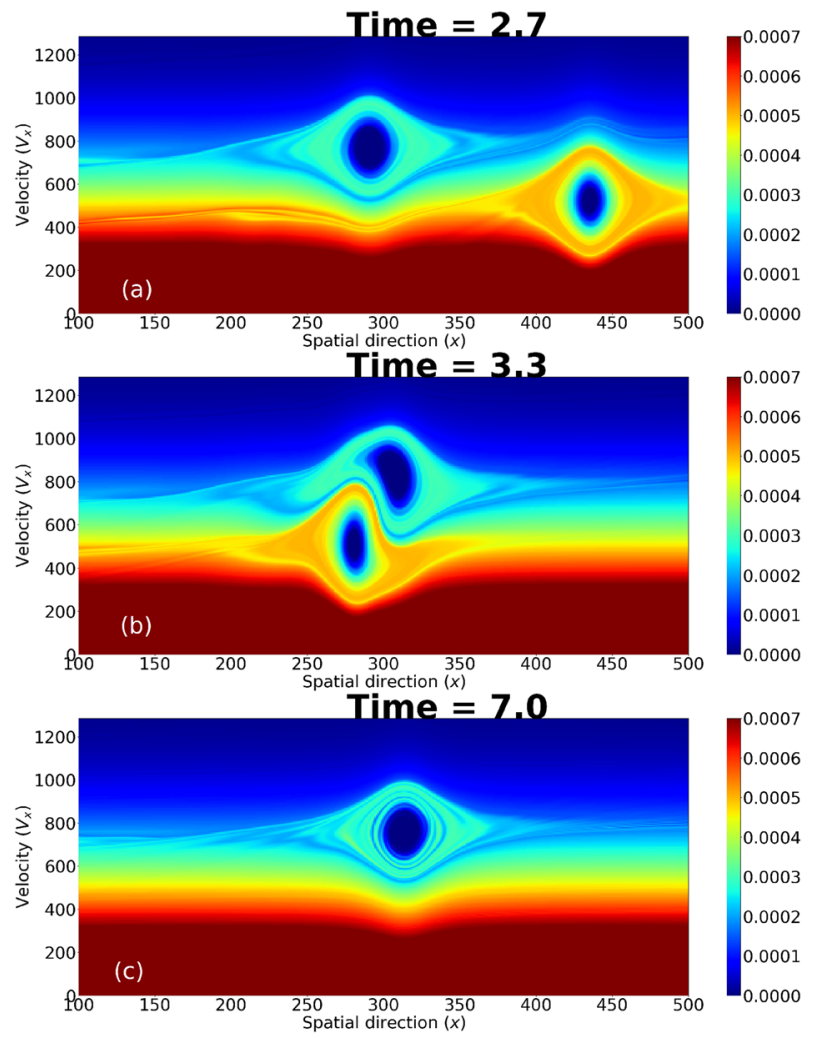

Figure 6. The electron phase space is presented for an overtaking collision between $E H 1$ and $E H 3$ in the co-moving frame of $E H 1$. There is a substantial overlap in velocity direction (a). During collision, the interaction is strong $(\mathbf{b})$. After the collision, $E H 1$ reappears un-altered in (c).

\section{Methods}

Equation set. The scaled Vlasov-Ampère system of equations forming the basis of our simulation reads:

$$
\begin{gathered}
\frac{\partial f_{s}(x, v, t)}{\partial t}+v \frac{\partial f_{s}(x, v, t)}{\partial x}+\Upsilon_{s} E(x, t) \frac{\partial f_{s}(x, v, t)}{\partial v}=0, \\
\frac{\partial E(x, t)}{\partial t}=\sum q_{s} J_{s}(x, t)
\end{gathered}
$$

where $s=i, e$ represents the corresponding species, i.e. ions and electrons respectively. The factor $\Upsilon_{s}$ takes the values $\Upsilon_{e}=-1836$ and $\Upsilon_{i}=1$. The normalized charges are $q_{e}=-1$ and $q_{i}=1$. The above equations are coupled by integrations for each species, viz. $J_{s}(x, t)=\int f_{s}(x, v, t) v d v$ in order to form a closed set of equations for $J$, denoting the current (contribution) generated by by species $s$. To derive the above (dimensionless) equations, all physical quantities were normalized to suitable scales related with ionic parameters, i.e. mass $\left(m_{s}\right)$ was divided by the ion mass $\left(m_{i}\right)$, temperature $\left(T_{s}\right)$ by ion temperature $\left(T_{i}\right)$, charge $\left(q_{s}\right)$ by the elementary charge $(e)$, time $(\tau)$ by the ion plasma period $\left(\omega_{p i}^{1 / 2}=\left(\frac{n_{i 0} e^{2}}{m_{i} \epsilon_{0}}\right)^{-\frac{1}{2}}\right)$, and length $(L)$ by the ion Debye length $\left(\lambda_{D i}=\sqrt{\frac{\epsilon_{0} K_{B} T_{i}}{n_{i 0} e^{2}}}\right)$. Here, $K_{B}$ is Boltzmann's constant and $\epsilon_{0}$ is the permittivity of free space.

Simulation code. We have employed the Gkeyll simulation framework ${ }^{33}$ to solve the Vlasov-Ampere system of equations ${ }^{34-36}$. Gkeyll discretizes the equations using the discontinuous Galerkin finite element method in space, with a strong stability-preserving Runge-Kutta method in time. We have adopted a piecewise cubic Serendipity Element space for the basis expansion ${ }^{37}$ (further details can be found in Refs. ${ }^{34,36}$ ). The Gkeyll method has been compared to the standard PIC method, where it was demonstrated that the effective phase space resolution of the method is very high, permitting detailed studies of df dynamics. Such high accuracy is of paramount importance for the resolution of EH dynamics in phase space ${ }^{38}$.

Parameters. In our study, the temperature and mass ratio are $\frac{T_{e}}{T_{i}}=100$ and $\frac{m_{i}}{m_{e}}=1836$. The initial distribution function $f_{0}$ is considered to be the Maxwellian $\mathrm{df}\left(=D_{m}\right)$. The size (length) of the simulation box is $l=1000$ in the $x$-direction. In the $v$ direction for each species, we have different limits: for the electrons we have $v=(-6,6) v_{t h_{e}}=(-2571,2571)$ and for the ions we have $v=(-10,10)$, where $v_{t h}=\sqrt{\frac{T_{e}}{T_{i}} \frac{m_{i}}{m_{e}}} \approx 428.5$ is the 
electron thermal velocity. The number of grid cells in each direction is $n_{X}=2000, n_{V}=1000$ for both electrons and ions. The time step $\mathrm{d} \tau \approx 10^{-5}$ is chosen in order to fulfill Courant-Friedrichs-Lewy (CFL) condition ${ }^{39,40}$.

The electron hole speed $\left(v_{E H}\right)$ is expressed by the "Mach number", which is defined as the ratio $M=\frac{v_{E H}}{c_{s}}$, where $c_{s}=\sqrt{1+\frac{\gamma_{e} T_{e}+\gamma_{i} T_{i}}{m_{i}}}$ is the ion sound speed. Assuming $\gamma_{e}=\gamma_{i}=3$ (heat capacity ratio), $T_{e}=100 T_{i}$ and $m_{i}=1$, the ion sound speed in our simulations is $c_{s}=\sqrt{304} \approx 17.43$.

Iterative method to find stable solutions. Our method follows the BGK method and starts by adopting an arbitrary function for the electrostatic potential $(\phi(x))$ and by choosing the value of the electron hole speed $\left(v_{E H}\right)$. We then use the ELIN df to produce the electron distribution function. Given that the potential profile provides the charge density $(\rho(x))$, and using the Schamel $\mathrm{df}$ for the ions to obtain $n_{i}(x)$, we then use the total charge density (profile) $n_{e}(x)=\rho(x)-n_{i}(x)$ as a "guiding equation" for the ELIN $\mathrm{df}$ and thus construct the electron hole. We have adopted, to start with, the simplest form of potential profile suggested for electron holes i.e. $\phi=A \operatorname{sech}^{p}(x / L)$ in which $p=2$ and $A$ and $L$ are the $\mathrm{EH}$ amplitude and length, respectively. The amplitude and length (values) are chosen randomly; however the system will damp/break the forced profile if it is not close-enough to a self- consistent nonlinear solution. The resulting electron hole may have different size and velocity, but with an iterative process, one can find the combination of $\{A, L\}$ for which the solution will be stable enough for a specific (chosen) velocity value. Since we are not aware of the nonlinear dispersion relation, i.e. a relationship between $\{A, L, M, p\}$ for the exact nonlinear solution(s), a sequence of trials is performed to iterate to the correct combination of $\{A, L, p\}$ for a given $M$. In the simulations presented here three electron holes were studied, e.g.

- $E H 1: M=45, A=19, L=22.5, p=2$

- $E H 2: M=-40, A=9.5, L=22.5, p=2$

- $E H 3: M=30, A=19, L=22.5, p=2$.

Elin DF method to construct electron holes. In order to explain our novel distribution function approach, firstly we need to represent the Schamel distribution function in energy-dependent format. Here we briefly discuss this, more details can be found in the reference ${ }^{19}$. Schamel approach devides the distribution function into two parts, namely free and trapped particles which are separated by a separatrix.

Focusing on the free particles, the following steps are taken to determine their distribution function $\left(f_{f}\right)$, assuming a pulse moving with a velocity $\left(v_{E H}\right)$ in the laboratory frame:

1. the shifted kinetic energy is found in the co-moving frame: $\varepsilon_{K_{s h}}^{\prime}=\left|\varepsilon_{K}^{\prime}-\varepsilon_{\phi}\right|$ where $\varepsilon_{\phi}=q \phi, \varepsilon_{K}^{\prime}=\frac{1}{2} \frac{m}{T} v^{\prime 2}$ and $v^{\prime}=v-v_{E H}$ is the velocity in the co-moving frame.

2. the shifted kinetic energy is calculated in the laboratory frame: $\varepsilon_{K_{s h}}=\frac{1}{2} \frac{m}{T} v_{s h}^{2}$ in which $v_{s h}=v_{s h}^{\prime}+v_{E H}$ and subsequently $v_{s h}^{\prime}=\operatorname{sign}\left(v^{\prime}\right) \sqrt{2 \varepsilon_{K_{s h}}^{\prime} / m}$.

Free particles fulfill the condition $\varepsilon_{K}^{\prime}>\varepsilon_{\phi}$. Note that, in order to calculate the $\mathrm{df}$ at point $v$, we use the $\mathrm{df}$ at the point $v_{s h}$, which can be written as $f=D_{g}\left(\varepsilon_{K_{s h}}\right)$ in energy format. Here, $v_{s h}$ presents the velocity of particles before their interaction with the potential profile. By $D_{g}$ we denote a general distribution function satisfying the Vlasov equation, i.e. in principle any function depending on the constant(s) of motion. Here, the energy is used to construct a valid function. Well-known examples of $D_{g}$ are the Maxwell-Boltzmann $\mathrm{df}$, the $\kappa \mathrm{df}^{41-44}$ and the Cairns ${ }^{45}$ distribution function(s).

In other words we trace the characteristics of the particle back in phase space. Then we use the value of $\mathrm{df}$ at $v_{s h}$ as the value of $\mathrm{df}$ for $v$ since the $\mathrm{df}$ stays constant on the characteristics of Vlasov equation ${ }^{46}$.

The distribution function of trapped particles $\left(f_{t}\right)$ which are subject to the trapping condition $\left(\varepsilon_{K}^{\prime}<\varepsilon_{\phi}\right)$ can be achieved by following the steps below:

1. the shifted kinetic energy is found in the co-moving frame: $\varepsilon_{K_{s h}}^{\prime}=\left|\varepsilon_{K}^{\prime}-\varepsilon_{\phi}\right|$, using a Maxwellian df on top of this kinetic energy with a coefficient $\beta$, will provide the shape of trapped distribution function: $f_{\text {shape }}=D_{m}\left(\beta \varepsilon_{K_{\text {sh }}}^{\prime}\right)=\exp \left(-\beta \varepsilon_{K_{\text {sh }}}^{\prime}\right)$

2. In order to have continuity between trapped and free $\mathrm{df}$ where they meet in the velocity direction, $f_{\text {shape }}$ is multiplied by $f_{\text {base }}=D_{g}\left(\varepsilon_{S}\right)$. Hence $f_{t}=f_{\text {base }} \times f_{\text {shape }}$.

Here, $D_{g}\left(\varepsilon_{S}\right)$ stands for the distribution function at the separatrix where $\varepsilon_{K}^{\prime}=\varepsilon_{\phi}$ and works as a constant value which can increase or decrese the $f_{t}$, in order to adjust it with the free distribution function. The second component, $f_{\text {shape }}$ is velocity-dependent and is controlled by $\beta$. It may appear in three qualitative shapes, i.e. flat, a bump or a hollow curve, if $\beta=0, \beta>0$ or $\beta<0$, respectively (see Fig. 1).

Hence, the total form of the Schamel distribution function ${ }^{12}$ can be written in terms of the energy as: $f=a f\left(\varepsilon_{K}\right)$ in which $a$ is a normalization constant and 


$$
f\left(\varepsilon_{K}\right)= \begin{cases}f_{f}=D_{g}\left(\varepsilon_{K_{s h}}\right) & \text { if } \varepsilon_{K}^{\prime}>\varepsilon_{\phi} \\ D_{g}\left(\varepsilon_{S}\right) & \text { if } \varepsilon_{K}^{\prime}=\varepsilon_{\phi} \\ f_{t}=D_{g}\left(\varepsilon_{S}\right) D_{m}\left(\beta\left|\varepsilon_{K}^{\prime}-\varepsilon_{\phi}\right|\right) & \text { if } \varepsilon_{K}^{\prime}<\varepsilon_{\phi}\end{cases}
$$

One can understand the Schamel $\mathrm{df}$ as carving up a given general distribution function $\left(D_{g}\right)$ around a particular velocity (hole velocity) and inserting a Maxwellian df with arbitrary temperature inside the hole $\left(f_{t}\right)$.

In the above representation of the Schamel df we used the analytical form of $\phi(x)$. However, one can equally use the discretizied form of $\phi(x)$ (by deviding it into a $n$ intervals of $\Delta \phi$ ). Schamel $\mathrm{df}$ can then be retrieved by $n \rightarrow \infty(\Delta \phi \rightarrow 0)$. In terms of simulation approach, these two methods are equal since even when using the analytical approach, one had to use discretization for $\phi(x)$ and there is limit on how small $\Delta \phi$ can get.

In other words, to generate distribution function $\left(f_{f}\right.$ and $\left.f_{t}\right)$ for each interval, we only need update the value of $f_{b a s e}$ in our approach and repeat the process. This results in multiple carvings, each based on the previous distribution function and it recursively progresses.

We assume $\phi=A \operatorname{sech}^{2}(x / L)$ as crude approach (stablished by trial and error in the beginning), and then we dicretize the first half of $\phi(x)$ into $n$ intervals in the following form $\left(\Delta \phi=\frac{A}{n}\right)$ :

$$
\begin{aligned}
\phi\left(x_{1}\right) & =\Delta \phi \\
\phi\left(x_{i}\right) & =\phi\left(x_{i-1}\right)+\Delta \phi \\
\phi\left(x_{n}=\frac{L}{2}\right) & =A .
\end{aligned}
$$

The second half will be the same as the first half except for a simple inversion. Hence we just build the first half of $\mathrm{df}$ and the second half is just simple inverted copy of it.

In this approach $\beta$ can be changed for each interval, and this add a new degree of freedom to the Schamel df. We call this ELIN (rEcursiveLy extendable distribution for a trapped populatioN) distribution function. The distribution function for each interval can be presented by the following equation. In which the $D_{g}$ (in Schamel df) is replaced by the distribution function $f_{i-1}$ of previous interval and each interval has its own $\beta_{i}$ :

$$
f\left(\phi_{i}\right)= \begin{cases}f_{f}=f_{i-1}\left(\varepsilon_{K_{s h}}\right) & \text { if } \varepsilon_{K}^{\prime}>q \Delta \phi_{i} \\ f_{i-1}\left(\varepsilon_{S}\right) & \text { if } \varepsilon_{K}^{\prime}=q \Delta \phi_{i} \\ f_{t}=f_{i-1}\left(\varepsilon_{S}\right) D_{m}\left(\beta_{i}\left(\left|\varepsilon_{K}^{\prime}-\varepsilon_{\phi}\right|\right)\right) & \text { if } \varepsilon_{K}^{\prime}<q \Delta \phi_{i}\end{cases}
$$

in which $f_{0}$ is the initial unperturbed $\mathrm{df}$ (here assuming Maxwellian df, i.e. $f_{0}=D_{m}$ ). $\beta_{i}$ can change arbitrarily in order for moments of df to fit a "guiding equation" (here, the equation for the electron density). To obtain a smooth distribution function in the $x$ direction, one can increase $n$ until the numerically-desired level of smoothness is achieved. An example of the ELIN df profile is presented at Fig. 1 which shows 10 successive (carving) iterations with $\beta$ approaching zero from below (negative side). Note that since $\beta$ originates from a continuous guiding equation, hence their successive values follow a pattern and are not randomly chosen.

To conclude, we have introduced a new method for constructing electron holes within a kinetic framework, which relies on a successive multi-step extension of the Schamel df (here represented in energy-dependent form), i.e. the ELIN df method. The ELIN df adopts a continuously varying value for $\beta$, in contrast to the Schamel df where $\beta$ is a constant. This extension provides an infinite number of parameters for the ELIN df, which enables it to construct an electron hole for any given bell-shaped potential profile. In our computational approach, the number of free parameters in the ELIN df is finite and equals the number of intervals $(n)$. We have adopted an iterative method (inspired by Newton's iterative scheme), built on top of the ELIN df method, to find the stable solutions. Starting from an initial guess, in each iteration of this method firstly we use the ELIN df to build an electron hole and then utilize the Vlasov-Poisson simulation method to follow the temporal evolution of the electron hole for a short time. We use the potential profile at the end of each iteration as an input for the next round of iteration. After a few iterations, the initial and final potential profiles are close enough for this to be considered as a stable configuration, for closure. Then, one can move on to longer-time numerical experiments, to investigate the long-time evolution of these localized structures and their behavior through mutual collisions. As a representative set, three stable solutions (i.e. EH1, EH2 and EH3; see above) have been reported in detail.

Received: 8 November 2020; Accepted: 16 June 2021

Published online: 11 August 2021

\section{References}

1. Schamel, H. Electron holes, ion holes and double layers: Electrostatic phase space structures in theory and experiment. Phys. Rep. 140, 161-191 (1986)

2. Schamel, H. Particle trapping: A key requisite of structure formation and stability of vlasov-poisson plasmas. Phys. Plasmas 22, 042301 (2015).

3. Hutchinson, I. H. Electron holes in phase space: What they are and why they matter. Phys. Plasmas 24, 055601 (2017).

4. Bernstein, I. B., Greene, J. M. \& Kruskal, M. D. Exact nonlinear plasma oscillations. Phys. Rev. 108, 546 (1957).

5. Saeki, K., Michelsen, P., Pécseli, H. \& Rasmussen, J. J. Formation and coalescence of electron solitary holes. Phys. Rev. Lett. 42, 501 (1979).

6. Ergun, R. et al. Fast satellite observations of large-amplitude solitary structures. Geophys. Res. Lett. 25, 2041-2044 (1998). 
7. Franz, J. R., Kintner, P. M. \& Pickett, J. S. Polar observations of coherent electric field structures. Geophys. Res. Lett. 25, 1277-1280 (1998).

8. Matsumoto, H. et al. Electrostatic solitary waves (esw) in the magnetotail: Ben wave forms observed by geotail. Geophys. Res. Lett. 21, 2915-2918 (1994).

9. Kojima, H. et al. Geotail waveform observations of broadband/narrowband electrostatic noise in the distant tail. J. Geophys. Res. Space Phys. 102, 14439-14455 (1997).

10. Deng, X. et al. Observations of electrostatic solitary waves associated with reconnection by geotail and cluster. Adv. Space Res. 37, 1373-1381 (2006).

11. Eliasson, B. \& Shukla, P. K. Formation and dynamics of coherent structures involving phase-space vortices in plasmas. Phys. Rep. 422, 225-290 (2006).

12. Schamel, H. Stationary solutions of the electrostatic Vlasov equation. Plasma Phys. 13, 491 (1971).

13. Schamel, H. Non-linear electrostatic plasma waves. J. Plasma Phys. 7, 1-12 (1972).

14. Schamel, H. Stationary solitary, snoidal and sinusoidal ion acoustic waves. Plasma Phys. 14, 905 (1972).

15. Schamel, H. Role of trapped particles and waves in plasma solitons-theory and application. Phys. Scr. 20, 306 (1979).

16. Schamel, H. Two-parametric, mathematically undisclosed solitary electron holes and their evolution equation. Plasma 3, 166-179 (2020).

17. Schamel, H. \& Bujarbarua, S. Analytical double layers. Phys. Fluids 26, 190-193 (1983).

18. Sagdeev, R. Cooperative phenomena and shock waves in collisionless plasmas. Rev. Plasma Phys. 4, 23 (1966).

19. Hosseini Jenab, S., Spanier, F. \& Brodin, G. A study of the stability properties of Sagdeev solutions in the ion-acoustic regime using kinetic simulations. Phys. Plasmas 25, 072304 (2018).

20. Hosseini Jenab, S. \& Brodin, G. Head-on collision of nonlinear solitary solutions to Vlasov-Poisson equations. Phys. Plasmas 26, 022303 (2019).

21. Turikov, V. Electron phase space holes as localized BGK solutions. Phys. Scr. 30, 73 (1984).

22. Bujarbarua, S. \& Schamel, H. Theory of finite-amplitude electron and ion holes. J. Plasma Phys. 25, 515-529 (1981).

23. Muschietti, L., Roth, I., Ergun, R. \& Carlson, C. Analysis and simulation of BGK electron holes. Phys. Fluids Nonlinear Process. Geophys. 6, 211-219 (1999).

24. Muschietti, L., Ergun, R., Roth, I. \& Carlson, C. Phase-space electron holes along magnetic field lines. Geophys. Res. Lett. 26, $1093-1096$ (1999).

25. Nishida, Y. \& Nagasawa, T. Oblique collision of plane ion-acoustic solitons. Phys. Rev. Lett. 45, 1626 (1980).

26. Verheest, F., Hellberg, M. A. \& Hereman, W. A. Head-on collisions of electrostatic solitons in nonthermal plasmas. Phys. Rev. E 86, $036402(2012)$.

27. Nakamura, Y., Bailung, H. \& Lonngren, K. Oblique collision of modified Korteweg-de Vries ion-acoustic solitons. Phys. Plasmas 6, 3466-3470 (1999).

28. Marchant, T. R. Asymptotic solitons for a higher-order modified Korteweg-de Vries equation. Phys. Rev. E 66, 046623 (2002).

29. Demiray, H. Interactions of nonlinear ion-acoustic waves in a collisionless plasma. J. Comput. Appl. Math. 206, 826-831 (2007).

30. Dubinov, A., Kolotkov, D. Y. \& Sazonkin, M. Supernonlinear waves in plasma. Plasma Phys. Rep. 38, 833-844 (2012).

31. Verheest, F., Hellberg, M. A. \& Kourakis, I. Electrostatic supersolitons in three-species plasmas. Phys. Plasmas (1994-present) 20, $012302(2013)$.

32. Saha, A., Chatterjee, P. \& Banerjee, S. An open problem on supernonlinear waves in a two-component Maxwellian plasma. Eur. Phys. J. Plus 135, 1-8 (2020).

33. Hakim, A. H. et al. Continuum electromagnetic gyrokinetic simulations of turbulence in the tokamak scrape-off layer and laboratory devices. Phys. Plasmas 27, 042304 (2020).

34. Juno, J., Hakim, A., TenBarge, J., Shi, E. \& Dorland, W. Discontinuous Galerkin algorithms for fully kinetic plasmas. J. Comput. Phys. 353, 110-147 (2018).

35. Hakim, A., Francisquez, M., Juno, J. \& Hammett, G. W. Conservative discontinuous Galerkin schemes for nonlinear DoughertyFokker-Planck collision operators. J. Plasma Phys. 86, 905860403. https://doi.org/10.1017/S0022377820000586 (2020).

36. Hakim, A., \& Juno, J. Alias-free, matrix-free, and quadrature-free discontinuous Galerkin algorithms for (plasma) kinetic equations. In SC20: International Conference for High Performance Computing, Networking, Storage and Analysis (pp. 1-15) IEEE. https://doi. org $/ 10.5555 / 3433701.3433798$ (2020).

37. Arnold, D. N. \& Awanou, G. The serendipity family of finite elements. Found. Comput. Math. 11, 337-344 (2011).

38. Juno, J., Swisdak, M. M., Tenbarge, J. M., Skoutnev, V. \& Hakim, A. Noise-induced magnetic field saturation in kinetic simulations. J. Plasma Phys. 86, 175860401. https://doi.org/10.1017/S0022377820000707 (2020).

39. Courant, R., Friedrichs, K. \& Lewy, H. Über die partiellen differenzengleichungen der mathematischen physik. Math. Ann. 100, 32-74 (1928).

40. Courant, R., Friedrichs, K. \& Lewy, H. On the partial difference equations of mathematical physics. IBM J. Res. Dev. 11, 215-234 (1967).

41. Vasyliunas, V. M. A survey of low-energy electrons in the evening sector of the magnetosphere with OGO 1 and OGO 3. J. Geophys. Res. 73, 2839-2884 (1968).

42. Pierrard, V. \& Lazar, M. Kappa distributions: theory and applications in space plasmas. Sol. Phys. 267, 153-174 (2010).

43. Summers, D. \& Thorne, R. M. The modified plasma dispersion function. Phys. Fluids B 3, 1835-1847 (1991).

44. Hellberg, M. A. et al. "Comment on "Mathematical and physical aspects of Kappa velocity distribution"[Phys. Plasmas 14, 110702 (2007)]." Phys. Plasmas 16(9), 094701. https://doi.org/10.1063/1.3213388 (2009).

45. Cairns, R. et al. Electrostatic solitary structures in non-thermal plasmas. Geophys. Res. Lett. 22, 2709-2712 (1995).

46. Kazeminezhad, F., Kuhn, S. \& Tavakoli, A. Vlasov model using kinetic phase point trajectories. Phys. Rev. E 67, 026704 (2003).

\section{Acknowledgements}

This project has received funding from the European Research Council (ERC) under the European Union's Horizon 2020 research and innovation programme under Grant Agreement No 647121. Simulations were performed on resources of High Performance Computing Center North (HPC2N) provided by the Swedish National Infrastructure for Computing (SNIC). J. Juno was supported by a NSF Atmospheric and Geospace Science Postdoctoral Fellowship (Grant No. AGS-2019828) and a NASA Earth and Space Science Fellowship (Grant No. 80NSSC17K0428). One of us (IK) gratefully acknowledges support from ADEK (Abu Dhabi Department of Education and Knowledge) in the form of an AARE-2018 (ADEK Award for Research Excellence 2018) grant. IK also acknowledges support from Khalifa University (internal funding) via Grant FSU-2021-012 (8474000352) (Modeling of Nonlinear Waves and Shocks in Space and Laboratory Plasmas). 


\section{Author contributions}

The main author (S.M.H.J.) has carried out the simulations and prepared the figures and suggested the theoretical framework of Elin DF. He also prepared the initial draft. I.K. rewrote and improved the manuscript. He also contributed greatly to the theoretical framework of the paper and enhanced it. G.B. edited and reviewed the manuscript and contributed greatly to understanding the results. J.J. (as the developer of Gkyll project) contributed in preparing the initial input file for Gkyll to run the simulations. He also edited the draft and wrote the paragraph about Gkyll code. All authors reviewed the manuscript.

\section{Funding}

Open access funding provided by Chalmers University of Technology.

\section{Competing interests}

The authors declare no competing interests.

\section{Additional information}

Correspondence and requests for materials should be addressed to S.M.H.J.

Reprints and permissions information is available at www.nature.com/reprints.

Publisher's note Springer Nature remains neutral with regard to jurisdictional claims in published maps and institutional affiliations.

(c) (i) Open Access This article is licensed under a Creative Commons Attribution 4.0 International License, which permits use, sharing, adaptation, distribution and reproduction in any medium or format, as long as you give appropriate credit to the original author(s) and the source, provide a link to the Creative Commons licence, and indicate if changes were made. The images or other third party material in this article are included in the article's Creative Commons licence, unless indicated otherwise in a credit line to the material. If material is not included in the article's Creative Commons licence and your intended use is not permitted by statutory regulation or exceeds the permitted use, you will need to obtain permission directly from the copyright holder. To view a copy of this licence, visit http://creativecommons.org/licenses/by/4.0/.

(C) The Author(s) 2021 